$1-1-2005$

\title{
Wave propagation in carbon nanotubes via nonlocal continuum mechanics
}

Q. Wang

University of Central Florida

Find similar works at: https://stars.library.ucf.edu/facultybib2000 University of Central Florida Libraries http://library.ucf.edu

This Article is brought to you for free and open access by the Faculty Bibliography at STARS. It has been accepted for inclusion in Faculty Bibliography 2000 s by an authorized administrator of STARS. For more information, please contact STARS@ucf.edu.

\section{Recommended Citation}

Wang, Q., "Wave propagation in carbon nanotubes via nonlocal continuum mechanics" (2005). Faculty Bibliography 2000s. 5755.

https://stars.library.ucf.edu/facultybib2000/5755

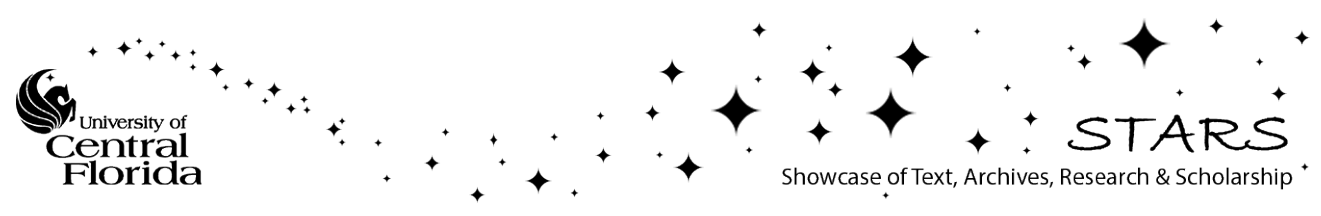




\section{Wave propagation in carbon nanotubes via nonlocal continuum mechanics}

Cite as: J. Appl. Phys. 98, 124301 (2005); https://doi.org/10.1063/1.2141648

Submitted: 22 April 2005 . Accepted: 04 November 2005. Published Online: 16 December 2005

Q. Wang

$\begin{array}{ll}10 & 0 \\ 5 & 0 \\ 5 & 0 \\ 0 & 0\end{array}$

\section{ARTICLES YOU MAY BE INTERESTED IN}

On differential equations of nonlocal elasticity and solutions of screw dislocation and surface waves

Journal of Applied Physics 54, 4703 (1983); https://doi.org/10.1063/1.332803

Column buckling of multiwalled carbon nanotubes using nonlocal continuum mechanics Journal of Applied Physics 94, 7281 (2003); https://doi.org/10.1063/1.1625437

Nonlocal continuum theories of beams for the analysis of carbon nanotubes

Journal of Applied Physics 103, 023511 (2008); https://doi.org/10.1063/1.2833431

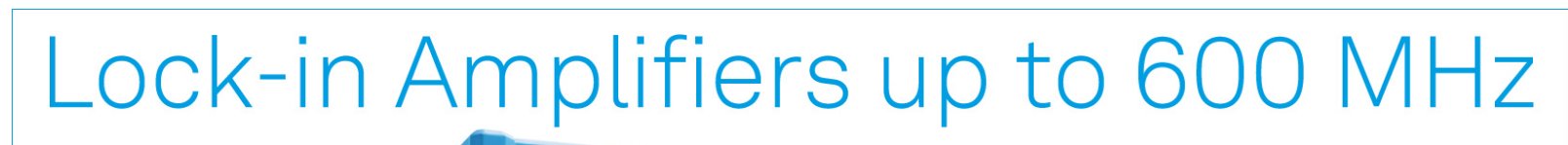

starting at

\section{$\$ 6,210$}

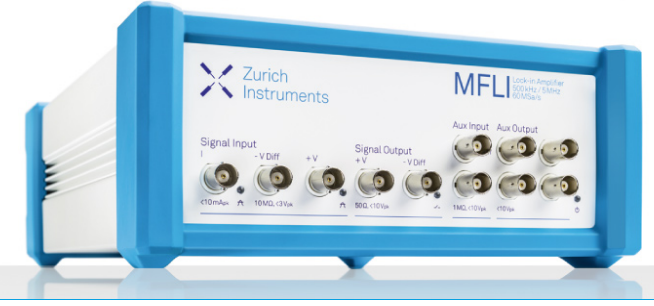




\title{
Wave propagation in carbon nanotubes via nonlocal continuum mechanics
}

\author{
Q. Wang ${ }^{\text {a) }}$ \\ Department of Mechanical, Materials and Aerospace Engineering, University of Central Florida, Orlando, \\ Florida 32816-2450
}

(Received 22 April 2005; accepted 4 November 2005; published online 16 December 2005)

\begin{abstract}
Wave propagation in carbon nanotubes (CNTs) is studied with two nonlocal continuum mechanics models: elastic Euler-Bernoulli and Timoshenko beam models [Philos. Mag. 41, 744 (1921)]. The small-scale effect on CNTs wave propagation dispersion relation is explicitly revealed for different CNTs wave numbers and diameters by theoretical analyses and numerical simulations. The asymptotic phase velocities and frequency are also derived from nonlocal continuum mechanics. The scale coefficient in nonlocal continuum mechanics is roughly estimated for CNTs from the obtained asymptotic frequency. In addition, the applicability and comparison of the two nonlocal elastic beam models to CNTs wave propagation are explored through numerical simulations. The research findings are proved effective in predicting small-scale effect on CNTs wave propagation with a qualitative validation study based on the published experimental reports in this field. (C) 2005 American Institute of Physics. [DOI: 10.1063/1.2141648]
\end{abstract}

\section{INTRODUCTION}

Carbon nanotube (CNT) has become one of the most promising materials for nanotechnology ${ }^{1-5}$ due to its distinct electronic and mechanical properties. CNTs have been applied in many devices, such as atomic-force microscope, field emitters, nanofillers for composite materials, nanoscale electronic devices, and frictionless nanoactuators, nanomotors, nanobearings, and nanosprings. ${ }^{6}$ In addition to experimental works, much theoretical analysis on CNTs mechanical behavior has been conducted. The modeling for theoretical analysis is classified into two main categories. One category is atomic modeling, which includes techniques such as classical molecular dynamics (MD), tight binding molecular dynamics, and density functional model. ${ }^{7-10}$ These atomic methods are only applicable to systems with a small number of molecules and atoms and therefore restrained only to small-scale modeling. The other category is classical continuum modeling which, unlike classical molecular dynamics, is practical and useful in analyzing CNTs for large-scale systems. Yakobson, Brabec, and Bernholc ${ }^{11}$ studied the unique features of fullernes and developed a continuum shell model for studying different instability patterns of a CNT under different compressive loads. $\mathrm{Ru}^{12,13}$ proposed CNTs buckling analysis with elastic shell models. Parnes and Chiskis $^{14}$ investigated elastic buckling of nano/fiberreinforced composites with elastic beam models. Krishnan et $a l .{ }^{15}$ estimated Young's modulus of singled-walled CNTs by observing their freestanding room-temperature vibrations in a transmission electron microscope. The nanotube dimensions and vibration amplitudes were measured from electron micrographs. It was assumed that the vibration modes were driven stochastically and were those of a clamped cantilever. Wang ${ }^{16}$ provided effective in-plane stiffness and bending rigidity of armchair and zigzag CNTs through an analysis of a representative volume element of graphene layer with con-

${ }^{a)}$ Electronic mail: QZWANG@MAIL.UCF.EDU tinuous elastic models. Wang et al. ${ }^{17}$ studied bending instability characteristics of double-walled carbon nanotubes (DWNTs) of various configurations using a hybrid approach. Their simulations showed that bending instability could take place through the formation of a single kink at the midpoint of a DWNT or two kinks, placed symmetrically about the midpoint, depending upon both the tube length and diameters.

Nanotechnology's small scale makes the applicability of classical or local continuum models, such as beam and shell models, questionable. Classical continuum models do not admit intrinsic size dependence in the elastic solutions of inclusions and inhomogeneities. At nanometer scales, however, size effects often become prominent, the cause of which needs to be explicitly addressed due to an increasing interest in the general area of nanotechnology. ${ }^{18}$ Sun and Zhang ${ }^{19}$ indicated the importance of a semicontinuum model in analyzing nanomaterials after pointing out the limitations of the applicability of classical continuum models to nanotechnology. In their semicontinuum model for nanostructured materials with platelike geometry, material properties were found completely dependent on the thickness of the plate structure contrary to classical continuum models. The modeling of such a size-dependent phenomenon has become an interesting research subject in this field. ${ }^{20-22}$ It is thus concluded that the applicability of classical continuum models at very small scales is questionable, since the material microstructure, such as lattice spacing between individual atoms, becomes increasingly important at small size and the discrete structure of the material can no longer be homogenenized into a continuum. Therefore, continuum models need to be extended to consider the scale effect in nanomaterial studies. This can be accomplished through proposing nonlocal continuum mechanics models.

Nonlocal elasticity theory ${ }^{23,24}$ was proposed to account for the scale effect in elasticity by assuming the stress at a reference point to be a function of strain field at every point in the body. This way, the internal size scale could be simply 
considered in constitutive equations as a material parameter. Only recently has the nonlocal elasticity theory been introduced to nanomaterial applications. Peddieson, Buchanan, and $\mathrm{McNitt}^{25}$ applied nonlocal elasticity to formulate a nonlocal version of the Euler-Bernoulli beam model and concluded that nonlocal continuum mechanics could potentially play a useful role in nanotechnology applications. Inspired by the above conclusion on the applicability of nonlocal elasticity to nanotechnology, nonlocal Euler-Bernoulli beam and shell models have been employed to study CNTs mechanical behaviors. Sudak ${ }^{26}$ studied the infinitesimal column buckling of multiwalled nanotubes (MWNTs), incorporating not only van der Waals forces but also the effects of small length scales. His results showed that as the small length scale increased in magnitude, the critical axial strain decreased. Zhang, Liu, and Wang ${ }^{27}$ applied a nonlocal multishell model to buckling analysis of MWNTs under axial compression. Their results showed that the small scale's effect on the axial buckling strain was related to buckling mode and the length of tubes. Zhang, Liu, and $\mathrm{Xie}^{28}$ also studied a nonlocal double-elastic beam model for free transverse vibrations of double-walled CNTs. It was demonstrated that natural frequencies and the associated amplitude ratios of the inner to outer tubes were dependent upon the small length scale.

Recently, growing interest in terahertz physics of nanoscale materials and devices ${ }^{29-31}$ has drawn more attention to CNTs phonon dispersion relation, especially in the terahertz frequency range. Yoon, $\mathrm{Ru}$, and Mioduchowski ${ }^{32,33}$ studied MWNTs wave propagation with a multiple-beam model. In the analysis of one-dimensional beamlike structures, two models are usually employed, namely, the Euler-Bernoulli and Timoshenko beam models. Both models assume that plane sections remain plane. However, in the Euler-Bernoulli beam model, the sections are assumed to remain perpendicular to the neutral axis, whereas this assumption is removed in the Timoshenko beam model ${ }^{34}$ to account for shear and rotary effects. The Euler-Bernoulli beam model normally provides over-estimated wave phase velocity at higher wave number, while the Timoshenko beam model provides more accurate wave solution even at higher frequency range, though it is more complicated than the Euler-Bernoulli beam model. Wang and Varadan ${ }^{35}$ studied CNTs wave characteristics via classical (local) beam models. Numerical simulations for wave solution in a CNT by a local Euler-Bernoulli beam model and a local Timoshenko beam model showed that the Euler-Bernoulli beam model was inappropriate on the frequency range of terahertz. It was concluded that the Timoshenko beam model should be employed in the analysis of CNTs wave motion, especially for the frequency range of terahertz. All the above investigations analyzed the feasibility of the two local beam models in the analysis of CNTs wave propagation on terahertz frequency range. However, the small-scale effect was never considered in published results. Since terahertz physics of nanoscale materials and devices are major concerns for CNTs wave characteristics, the small-scale effect must be considered as the wavelength if the frequency domain is of the order of nanometer. This research tries to apply nonlocal continuum modeling to study CNTs wave characteristics. Nonlocal Euler-Bernoulli and Ti- moshenko elastic beam models are proposed. Explicit dispersive solutions are derived in the research, from which smallscale effects can be clearly observed. Such observations are vital for applying continuum models to obtain CNTs wave characteristics.

\section{NONLOCAL CONTINUUM MODELS OF CNTS}

In nonlocal elasticity, ${ }^{24}$ the stress at a reference point $x$ is considered to be a function of the strain field at every point in the body. This theory is in accordance with the atomic model of lattice dynamics and experimental observations on phonon dispersion. When the effect of strain at points other than $x$ is neglected, one obtains the local model of elasticity.

The basic equations for linear, homogeneous, isotropic, nonlocal elastic solid with zero body force are given $\mathrm{as}^{23}$

$$
\begin{aligned}
& \sigma_{i j, j}=0 \\
& \sigma_{i j}(x)=\int \alpha\left(\left|x-x^{\prime}\right|, \tau\right) C_{i j k l} \epsilon_{k l}\left(x^{\prime}\right) d V\left(x^{\prime}\right), \quad \forall x \in V, \\
& \epsilon_{i j}=\frac{1}{2}\left(u_{i, j}+u_{j, i}\right),
\end{aligned}
$$

where $C_{i j k l}$ is the elastic modulus tensor of classical isotropic elasticity, $\sigma_{i j}$ and $\epsilon_{i j}$ are stress and strain tensors, respectively, and $u_{i}$ is the displacement vector. $\alpha\left(\left|x-x^{\prime}\right|, \tau\right)$ is the nonlocal modulus or attenuation function which incorporates into the constitutive equations the nonlocal effects at the reference point $x$ produced by local strain at the source $x^{\prime}$. $\left|x-x^{\prime}\right|$ is Euclidean distance. In $\tau=e_{0} a / l,{ }^{23} e_{0}$ is a constant appropriate to each material, $a$ is an internal characteristic length (e.g., length of $\mathrm{C}-\mathrm{C}$ bond, lattice parameter, granular distance), and $l$ is an external characteristic length (e.g., crack length, wavelength). The value of $e_{0}$ needs to be determined from experiments or by matching dispersion curves of plane waves with those of atomic lattice dynamics.

Integral-partial differential equations of the above linear nonlocal elasticity are reduced to singular partial differential equations of a special class of physically admissible kernel. ${ }^{24}$ In addition, Hook's law for uniaxial stress state is determined by

$$
\sigma(x)-\left(e_{0} a\right)^{2} \frac{d^{2} \sigma(x)}{d x^{2}}=E \epsilon(x),
$$

where $E$ is the Young's modulus of the material. The parameter $e_{0} a$ is the scale coefficient revealing a small-scale effect on the response of structures in nanosize.

To investigate the small-scale effect on CNTs wave solutions, nonlocal Euler-Bernoulli and Timoshenko beam models for vibration analysis will be used.

\section{A. Nonlocal Euler-Bernoulli beam model}

The equilibrium of forces in the vertical direction and the moment on a free body diagram of an infinitesimal element of a beam structure is given as 


$$
\begin{aligned}
& \frac{\partial V}{\partial x}-\rho A \frac{\partial^{2} u}{\partial t^{2}}=0, \\
& V-\frac{\partial M}{\partial x}=0,
\end{aligned}
$$

where $V(x, t)$ and $M(x, t)$ are the resultant shear force and bending moment respectively, $\rho$ is the mass density of the material, $A$ is the area of the cross section, and $u(x, t)$ is the flexural deflection of the beam.

Considering definitions of the resultant bending moment and the kinematics relation in a beam structure, we have

$$
\begin{aligned}
& M=\int_{A} y \sigma d A, \\
& \epsilon=-y \frac{\partial^{2} u}{\partial x^{2}},
\end{aligned}
$$

where $y$ is the coordinate measured from the midplane along the direction of the beam's height.

Substituting Eqs. (4a) and (4b) into the nonlocal constitutive relation Eq. (2) leads to

$$
M-\left(e_{0} a\right)^{2} \frac{\partial^{2} M}{\partial x^{2}}=-E I \frac{\partial^{2} u}{\partial x^{2}},
$$

where $E I$ is the bending rigidity of the beam structure.

Further consideration of Eqs. (3a) and (3b), Eq. (5) gives

$$
E I \frac{\partial^{4} u}{\partial x^{4}}+\rho A \frac{\partial^{2}}{\partial t^{2}}\left(u-\left(e_{0} a\right)^{2} \frac{\partial^{2} u}{\partial x^{2}}\right)=0 .
$$

From Eq. (6), it is easily seen that the local EulerBernoulli beam model is recovered when the parameter $e_{0}$ is set to zero.

\section{B. Nonlocal Timoshenko beam model}

In the Timoshenko beam model, a new variable $\varphi$ is introduced to measure the slope of the cross section due to bending. The slope of the centroidal axis $\partial u / \partial x$ is constituted by $\varphi$, due to the bending effect, and $\gamma_{0}$, due to the shear effect. The essence of Timoshenko's argument is that the shear force at the cross section is expressed in terms of the shear strain $\gamma$ as

$$
V=G \int_{A} \gamma d A,
$$

where $G=E /[2(1+v)]$ is the shear modulus of CNTs. If $\gamma_{0}$ is the shear strain at the centroidal axis, $G \gamma_{0} A$ gives a shear force. However, it will not be equal to the value given in Eq. (7). To bring the value into balance, the adjustment coefficient $\kappa$ is introduced such that

$$
V=G \int_{A} \gamma d A=\kappa\left(G \gamma_{0} A\right)=A G \kappa\left(\frac{\partial u}{\partial x}-\phi\right) .
$$

The recommended value of $\kappa$, the adjustment coefficient, is $10 / 9$ for a circular shape of the cross area. ${ }^{34}$
From a similar analysis of the free body diagram, the equation of motion of CNTs based on the nonlocal Timoshenko beam model is given as

$$
\begin{aligned}
& \frac{\partial V}{\partial x}=\rho A \frac{\partial^{2} u}{\partial t^{2}}, \\
& V-\frac{\partial M}{\partial x}=\rho I \frac{\partial^{2} \phi}{\partial t^{2}},
\end{aligned}
$$

where the term on the right-hand side of Eq. (9b) is the rotary effect considered in the Timoshenko beam model.

Substitution of Eqs. (5) and (8) into Eqs. (9a) and (9b) leads to the following nonlocal Timoshenko beam model:

$$
\begin{gathered}
G A \kappa\left(\frac{\partial \varphi}{\partial x}-\frac{\partial^{2} u}{\partial x^{2}}\right)+\rho A \frac{\partial^{2} u}{\partial t^{2}}=0, \\
G A \kappa\left(1-\left(e_{0} a\right)^{2} \frac{\partial^{2}}{\partial x^{2}}\right)\left(\frac{\partial u}{\partial x}-\varphi\right)+E I \frac{\partial^{2} \varphi}{\partial x^{2}} \\
-\rho I \frac{\partial^{2}}{\partial t^{2}}\left(\varphi-\left(e_{0} a\right)^{2} \frac{\partial^{2} \varphi}{\partial x^{2}}\right)=0 .
\end{gathered}
$$

Again, it is seen that the local Timoshenko beam model is recovered ${ }^{34}$ when the parameter $e_{0}$ is identically zero.

\section{WAVE PROPAGATION IN CNTS BY NONLOCAL CONTINUUM MECHANICS}

The solution for wave propagation in CNTs will be derived via nonlocal Euler-Bernoulli and Timoshenko beam models hereinafter.

\section{A. Nonlocal Euler-Bernoulli beam model}

The wave propagation solution for Eq. (6) can be expressed as

$$
u(x, t)=U e^{i(k x-\omega t)},
$$

where $U$ is the amplitude of the wave motion, $k$ is the wave number, and $\omega$ is the frequency of the wave motion.

Substitution of Eq. (11) into Eq. (6) yields the solution for phase velocity via nonlocal the Euler-Bernoulli beam model

$$
\frac{\nu_{n E}}{\nu_{l E}}=\frac{1}{\sqrt{1+\left(e_{0} a\right)^{2} k^{2}}},
$$

where $\nu_{l E}=k \sqrt{E I / \rho A}$ is the wave phase velocity based on the local Euler-Bernoulli beam model.

\section{B. Nonlocal Timoshenko beam model}

Since an additional variable $\varphi$ is introduced in the Timoshenko beam model, the wave solution for this variable is given as

$$
\varphi(x, t)=\Phi e^{i(k x-\omega t)},
$$

where $\Phi$ is the amplitude of the wave motion.

Substitution of Eqs. (11) and (13) into Eqs. (10a) and (10b) leads to the following two equations: 


$$
\begin{aligned}
& \left(-\rho A \omega^{2}+G A k^{2} \kappa\right) U+i G A k \kappa \Phi=0, \\
& i G A k \kappa\left[1+\left(e_{0} a\right)^{2} k^{2}\right] U+\left(-E I k^{2}-G A \kappa\left[1+\left(e_{0} a\right)^{2} k^{2}\right]\right. \\
& \left.\quad+\rho I \omega^{2}\left[1+\left(e_{0} a\right)^{2} k^{2}\right]\right) \Phi=0 .
\end{aligned}
$$

From Eqs. (14a) and (14b), the solution for the wave propagation in CNTs via the nonlocal Timoshenko beam model can be derived from an eigen-value problem searching for nontrivial solution of the variables of $U$ and $\Phi$. The wave solution based on the nonlocal Timoshenko beam model, ignoring rotary effect, is thus obtained as

$$
\frac{\nu_{n T}}{\nu_{l T}}=\frac{1}{\sqrt{1+\left(e_{0} a\right)^{2} k^{2} \alpha}},
$$

where $\alpha=\left(1+d^{2} k^{2} / 8\right) /\left[1+d^{2} k^{2} / 8+(1+v) d^{2} k^{2} / 4 \kappa\right], d$ is the diameter of the midsurface of the CNT, $v$ is the Poisson's ratio, and $\nu_{l T}=\sqrt{(E I / \rho A) /\left(E I / G A \kappa+1 / k^{2}+\rho I / \rho A\right)}$ is CNTs phase velocity based on the local Timoshenko beam model.

\section{SIMULATIONS AND DISCUSSIONS}

The asymptotic phase velocity at $k \rightarrow \infty$ based on the nonlocal Euler-Bernoulli beam model is studied first. From Eq. (12), the asymptotic velocity is obtained as

$$
\bar{\nu}_{n E}=\frac{1}{e_{0} a} \sqrt{\frac{E I}{\rho A}} .
$$

The bar on the symbol in Eq. (16) stands for the asymptotic velocity. By considering the expressions for CNTs material parameters, ${ }^{35}$ the asymptotic velocity based on the local Timoshenko beam model can be obtained as

$$
\bar{\nu}_{l T}=\frac{1}{\sqrt{E I / G A \kappa+\rho I / \rho A}} \sqrt{\frac{E I}{\rho A}}=\frac{1}{d \sqrt{\frac{1}{8}+\frac{1+v}{4 \kappa}}} \sqrt{\frac{E I}{\rho A}} .
$$

From comparison of Eqs. (16) and (17), it is concluded that the nonlocal Euler-Bernoulli beam model is identical to the local Timoshenko beam model if the scale coefficient $e_{0} a$ is chosen as

$$
e_{0} a=d \sqrt{\frac{1}{8}+\frac{1+v}{4 \kappa}} .
$$

Natural logarithmic calculations of the phase velocity versus natural logarithmic calculations of the wave number of a CNT with $d=5 \mathrm{~nm}$, based on local and nonlocal EulerBernoulli beam models, respectively, are shown in Fig. 1 at different values of the scale coefficients, namely, $e_{0} a=0.5,1$, and $1.5 \mathrm{~nm}$. It is seen that at lower wave numbers $k<e^{20}$ $\approx 4 \times 10^{8} / \mathrm{m}$, or at the corresponding higher wavelength $\lambda$ $=1 / k>2.5 \mathrm{~nm}$, both beam models provide almost the same solution for the given domain of scale coefficient $e_{0} a$ $\leqslant 1.5 \mathrm{~nm}$. The local Euler-Bernoulli beam model shows the linear relation between the two variables, whereas the nonlocal Euler-Bernoulli beam model displays virtually nondispersive wave characteristic, i.e., constant phase wave veloc-

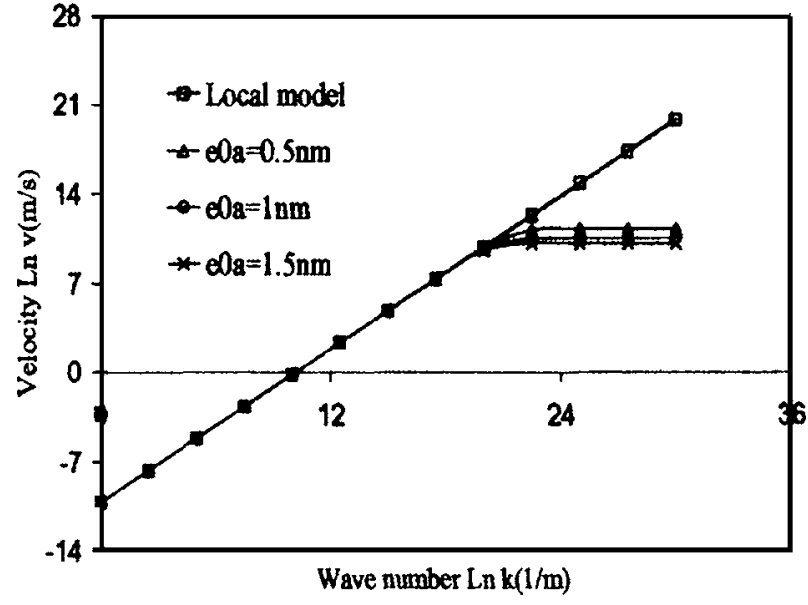

FIG. 1. Wave velocity versus wave number by Euler-Bernoulli model.

ity at higher frequency range or higher wave numbers, i.e., $k>4 \times 10^{8} / \mathrm{m}$ or $\lambda<2.5 \mathrm{~nm}$. The nondispersive phase velocity decreases at higher scale coefficient.

Figure 2(a) gives a comparison of the CNTs phase velocities based on local and nonlocal Timoshenko beam models and the nonlocal Euler-Bernoulli beam model at $e_{0} a$ $=3.35 \mathrm{~nm}$. The phase velocity from nonlocal beam model shows a decreasing variation with higher scale coefficient
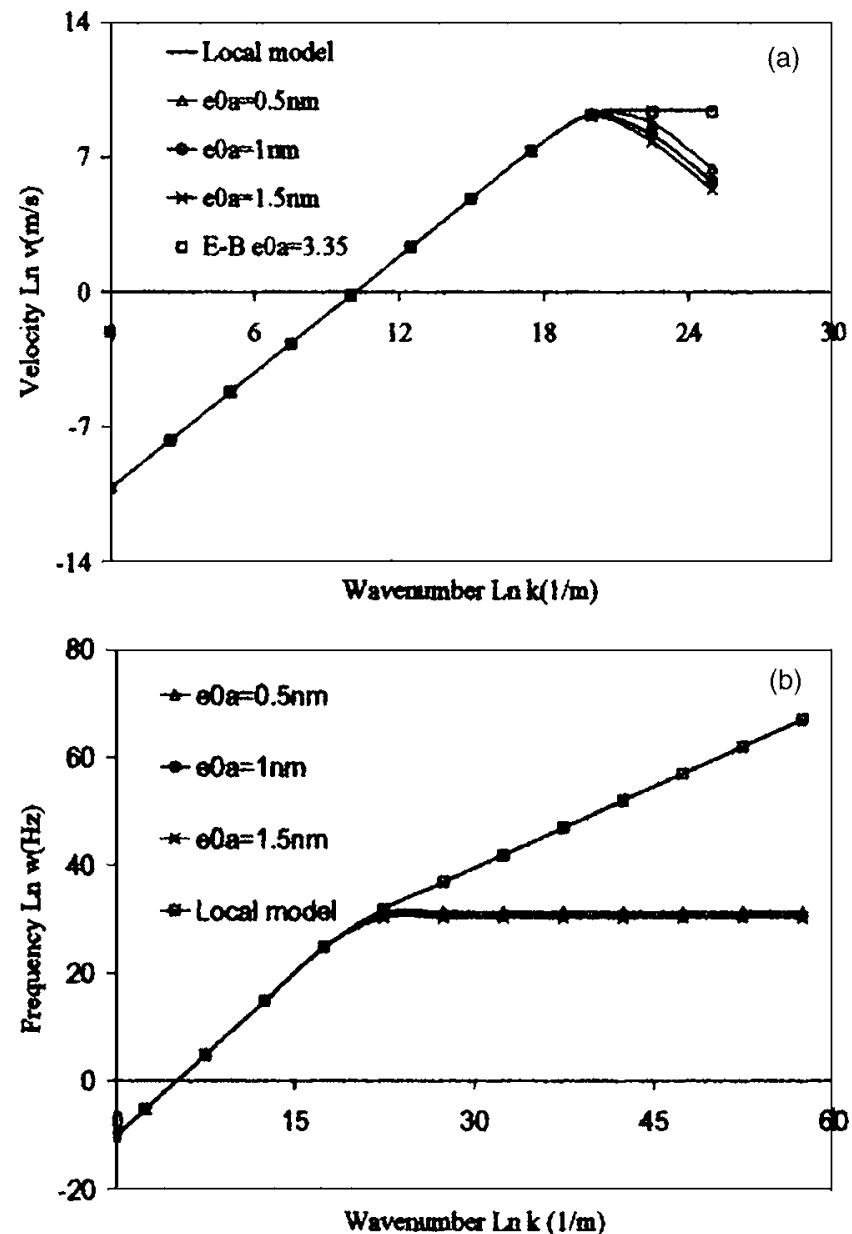

FIG. 2. Wave characteristics by Timoshenko model (a) wave velocity versus wave number and (b) wave frequency vs wave number. 
$e_{0} a$ similar to Fig. 1. In addition, the same solution is obtained at lower wave numbers $k<4 \times 10^{8} / \mathrm{m}$ or higher wavelength $\lambda>2.5 \mathrm{~nm}$ for the small-scale coefficient $e_{0} a$ $\leqslant 1.5 \mathrm{~nm}$. A comparison of Figs. 1 and 2(a) reveals an interesting observation that below the critical wave numbers $k$ $\approx 4 \times 10^{8} / \mathrm{m}$, or above the corresponding wavelength $\lambda$ $\approx 2.5 \mathrm{~nm}$, all local and nonlocal Euler-Bernoulli and Timoshenko beam models provide similar wave characteristic solutions for the CNT with $d=5 \mathrm{~nm}$ on the given scale coefficient domain $e_{0} a \leqslant 1.5 \mathrm{~nm}$. From Eq. (18), it is found that at $e_{0} a=3.35 \mathrm{~nm}$, for the CNT with $d=5 \mathrm{~nm}$, the nonlocal Euler-Bernoulli beam model provides the same solution for the phase velocity with the local Timoshenko beam model does. This is clearly observed in Fig. 2(a) from the concurrence of the curves from the local Timoshenko model and the nonlocal Euler-Bernoulli model at $e_{0} a=3.35 \mathrm{~nm}$. Since the Timoshenko model accounts for shear effect in modeling, it is recommended in the deriving wave solution, especially for a solution at a higher wave number or a smaller wavelength.

Figure 2(b) shows frequency comparison between local and nonlocal Timoshenko beam models. A linear variation of natural logarithmic frequency value versus natural logarithmic wave number is found for the local Timoshenko model. Further, a constant value of the frequency at higher wave number is observed for the nonlocal Timoshenko model. This constant value decreases with high scale coefficient $e_{0} a$. The asymptotic value of frequency can be derived from Eq. (15) as

$$
\bar{\omega}_{n T}=\frac{1}{e_{0} a} \sqrt{\frac{E t}{\rho t}}=\frac{1}{e_{0} a} \sqrt{\frac{C}{\rho t}},
$$

where in-plane stiffness $E t=C=360 \mathrm{~J} / \mathrm{m}^{2},{ }^{11}$ is virtually a constant parameter. The asymptotic frequency can thus be written as $\bar{\omega}_{n T}=21456 / e_{0} a$, if the mass density $\rho$ $=2.3 \mathrm{~g} / \mathrm{cm}^{3}$ and the thickness of CNT $t=0.34 \mathrm{~nm}$ are chosen. $^{33}$

Parameter $a$ describes internal characteristic length. The length of a C-C bond, which is $0.142 \mathrm{~nm}$, is chosen for the analysis of CNTs. ${ }^{25,26}$ On the other hand, parameter $e_{0}$ was given as 0.39 by Eringen. ${ }^{24}$ This value needs to be further verified through experiments or through matching dispersion curves of plane waves with those of atomic lattice dynamics for CNTs. It was speculated ${ }^{26}$ to be in the order of hundreds. Based on Eq. (19), a rough estimate of the scale coefficient $e_{0} a$ becomes possible as long as the highest frequency of a single-walled CNT can be available since vibration of a limit medium can be seen as a standing wave phenomenon in the medium. An available experimental work on the vibration of CNTs in Ref. 15 gives the fundamental frequency as around $0.1 \mathrm{THz}$. If this value is substituted in Eq. (19), $e_{0} a$ $<210 \mathrm{~nm}$ can be derived. As stated in Refs. 5 and 33, the frequency in CNTs is in the terahertz range. A conservative evaluation on the scale coefficient can be obtained as $e_{0} a$ $<2.1 \mathrm{~nm}$ for a SWNT if the measured frequency value for the SWNT is assessed to be greater than $10 \mathrm{THz}$. In addition, this value is radius dependent since frequency is a radius dependent parameter.

Figures 3 and 4 further demonstrate the conclusion that phase velocity decreases as scale coefficient and wave num-

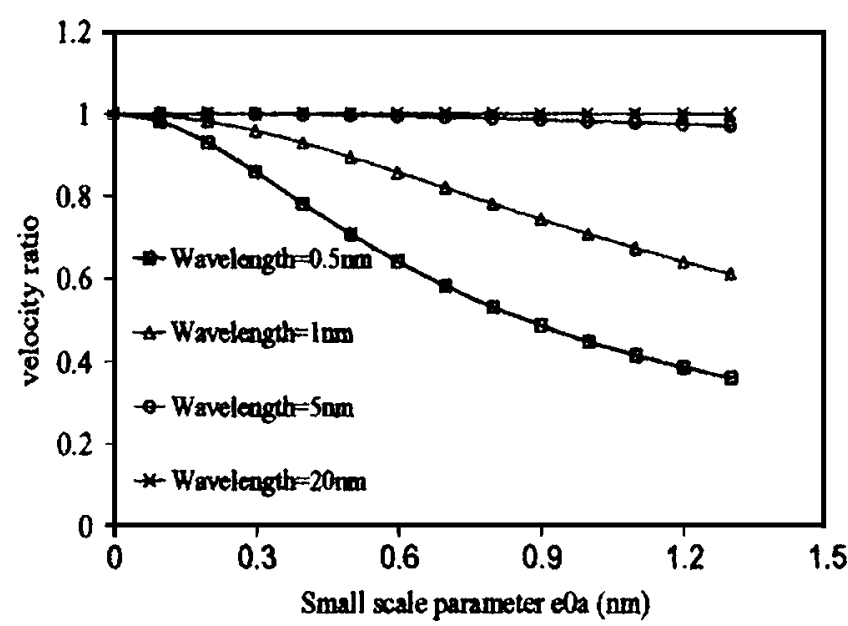

FIG. 3. Small effect on wave velocity by Euler-Bernoulli model.

ber increase. In the two figures, the vertical axis represents the ratio of the phase velocity from the nonlocal beam model to that from the local beam model. The variations of phase velocity versus the scale coefficient $e_{0} a(0-1.5 \mathrm{~nm})$ by Euler-Bernoulli and Timoshenko beam models are shown in the two figures, respectively. It can be observed that the velocity the ratio reaches unit at $e_{0} a=0$, from all the models. At higher wavelength, e.g., $1 / k=20 \mathrm{~nm}$, the ratio is seen to virtually approach unit.

In Fig. 5, the ratio of the phase velocity from the nonlocal Timoshenko beam model to that from the local Timoshenko beam model versus the diameter of the CNT at $e_{0} a$ $=1.5 \mathrm{~nm}$ is plotted. The four curves represent the variation of the velocity ratio at wavelength $\lambda=0.5,1,5$, and $20 \mathrm{~nm}$. It is clearly seen that the velocity ratio is lower at smaller diameters. Therefore, it can be concluded that CNTs wave solutions are diameter dependent based on nonlocal continuum mechanics. The small-scale effect on diameter becomes almost unnoticeable at larger diameters. This observation is distinct and interesting since preliminary research on the small-scale effect on buckling of $\mathrm{CNTs}^{26,27}$ has identified only length dependency. In addition, the velocity ratio is found to be lower at smaller wavelength.

From the numerical simulations, it can be concluded that at lower wavelength and smaller diameters, obvious smallscale effect is observed on CNTs wave characteristic solution

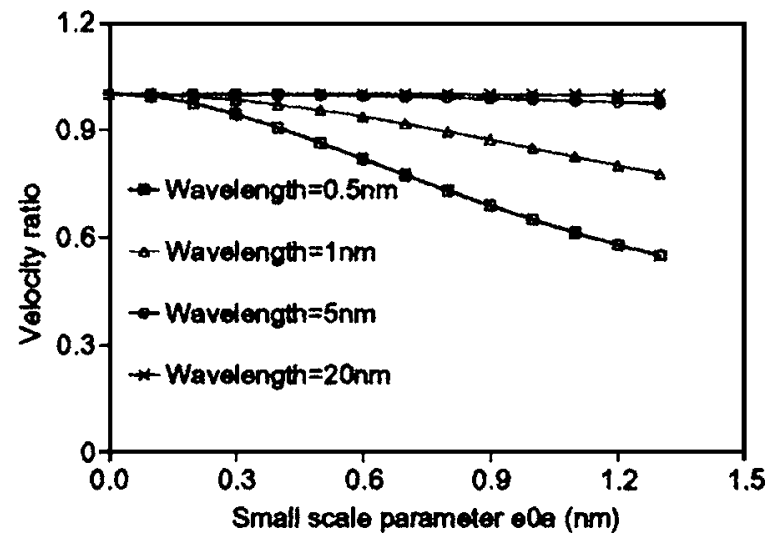

FIG. 4. Small effect on wave velocity by Timoshenko model. 


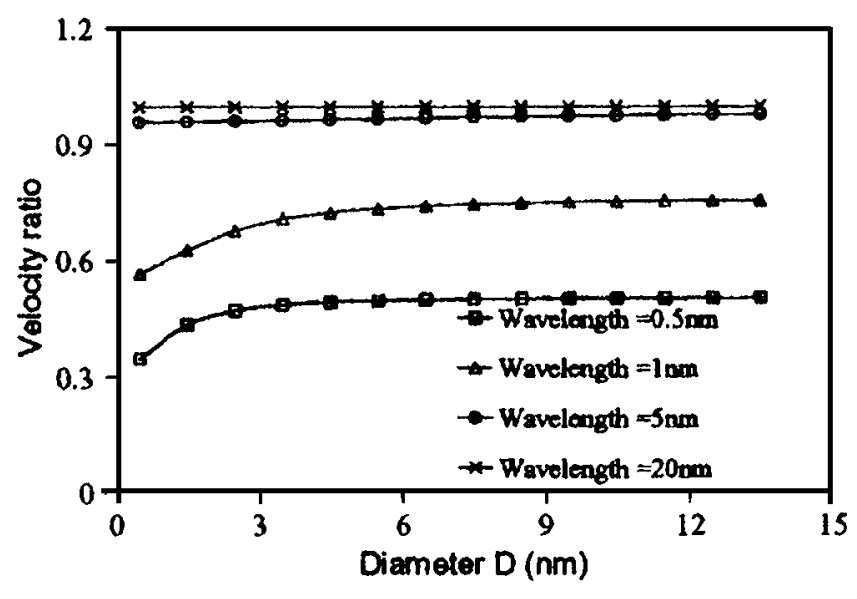

FIG. 5. Wave velocity versus diameter at $e_{0} a=1.5 \mathrm{~nm}$ by Timoshenko model.

via nonlocal continuum models. These observations are qualitatively in agreement with the experimental work by Krishnan et al. ${ }^{15}$ In their work, the stiffness of CNTs was estimated by observing their freestanding room temperature vibration in a transmission electron microscope. Independent estimates of the nanotube length and tip vibration amplitude were obtained from images of the cleanest nanotubes by a least-squares minimization procedure. The nanotube parameters, including nanotube length, width, and corresponding estimate of the Young's modulus were (a) $L=36.8 \mathrm{~nm}, W$ $=1.50 \mathrm{~nm}, E=1.33 \pm 0.2 \mathrm{TPa}$; (b) $L=24.3 \mathrm{~nm}, W=1.52 \mathrm{~nm}$, $E=1.20 \pm 0.2 \mathrm{TPa} ;$ (c) $L=23.4 \mathrm{~nm}, W=1.12 \mathrm{~nm}$, and $E$ $=1.02 \pm 0.3 \mathrm{TPa}$. The estimate of Young's modulus from their work was based on a classical frequency equation in local continuum mechanics from which the frequency is proportional to the square root of Young's modulus. Data in groups (a) and (b) show the comparison of the derived Young's modulus, or equivalently the frequency, of two CNTs at different lengths but with similar diameters. Since vibration of a finite medium can be seen as a standing wave phenomenon in the medium, the length of the medium is proportional to the wavelength of wave propagation in the medium. The frequency $\omega$ is related to phase velocity and wave number via the relation $L n \omega=L n \nu+L n k$. Therefore, the comparison of data in groups (a) and (b) indicates that the frequency or the phase wave velocity at a specific diameter is wavelength dependent. Lower frequency can be derived at smaller length or smaller wavelength of the CNT, which is qualitatively in agreement with the simulation results. In addition, from the comparison of data in groups (b) and (c), in which CNTs are with different diameters but with similar lengths, it can be seen that the derived frequency is lower at smaller diameters. This experimental result is again qualitatively in agreement with the simulations derived from nonlocal continuum models.

\section{CONCLUDING REMARKS}

In this research, nonlocal continuum models are applied in the analysis of CNTs wave characteristics. Nonlocal Euler-
Bernoulli and Timoshenko beam models enable the investigation of small-scale effect on a CNT's dispersion solutions. Wavelength and diameter-dependent results are explicitly derived from nonlocal continuum models. Extant experimental work validates the research findings. The research provides a rough estimation of the scale coefficient used in nonlocal continuum models. The work in the manuscript not only reveals the significance of the small-scale effect on CNTs mechanical response, but also points out the limitation of the applicability and feasibility of local continuum models in analysis of CNTs mechanical behaviors. Other nonlocal continuum models, such as elastic shell models, need to be studied thoroughly in order to gain a better understanding of CNTs mechanical behaviors.

${ }^{1}$ P. Ball, Nature (London) 414, 142 (2001).

${ }^{2}$ R. H. Baughman, A. A. Zakhidov, and W. A. de Heer, Science 297, 787 (2002).

${ }^{3}$ M. M. J. Treacy, T. W. Ebbesen, and T. M. Gibson, Nature (London) 381, 680 (1996).

${ }^{4}$ P. J. F. Harris, Carbon Nanotubes and Related Structures (Cambridge University Press, UK, 1999).

${ }^{5}$ S. Iijima, Nature (London) 354, 56 (1991).

${ }^{6}$ K. T. Lau, Chem. Phys. Lett. 370, 399 (2003).

${ }^{7}$ S. Iijima, C. Brabec, A. Maiti, and J. Bernholc, Chem. Phys. 104, 2089 (1996).

${ }^{8}$ B. I. Yakobson, M. P. Campbell, C. J. Brabec, and J. Bernholc, Comput. Mater. Sci. 8, 241 (1997).

${ }^{9}$ E. Hernandez, C. Goze, P. Bernier, and A. Rubio, Phys. Rev. Lett. 80, 4502 (1998).

${ }^{10}$ D. Sanchez-Portal et al., Phys. Rev. B 59, 12678 (1999).

${ }^{11}$ B. I. Yakobson, C. J. Brabec, and J. Bernholc, Phys. Rev. Lett. 76, 2511 (1996).

${ }^{12}$ C. Q. Ru, Phys. Rev. B 62, 9973 (2000).

${ }^{13}$ C. Q. Ru, Phys. Rev. B 62, 10405 (2000).

${ }^{14}$ R. Parnes and A. Chiskis, J. Mech. Phys. Solids 50, 855 (2002).

${ }^{15}$ A. Krishnan, E. Dujardin, T. W. Ebbesen, P. N. Yianilos, and M. M. J. Treacy, Phys. Rev. B 58, 14013 (1998).

${ }^{16}$ Q. Wang, Int. J. Solids Struct. 41, 5451 (2004).

${ }^{17}$ Q. Wang, T. Hu, G. Chen, and Q. Jiang, Phys. Rev. B 71, 045403 (2005).

${ }^{18}$ P. Sharma, S. Ganti, and N. Bhate, Appl. Phys. Lett. 82, 535 (2003).

${ }^{19}$ C. T. Sun and H. Zhang, J. Appl. Phys. 93, 1212 (2003).

${ }^{20}$ P. E. Sheehan and C. M. Lieber, Science 272, 1156 (1996).

${ }^{21}$ B. I. Yakobson and R. Smalley, Am. Sci. 85, 324 (1997).

${ }^{22}$ M. Terrones, N. Grobert, W. Hsu, Y. Hu, J. Terrones, H. Kroto, and D. Ealton, Mater. Res. Bull. 24, 43 (1999).

${ }^{23}$ A. C. Eringen, Nonlocal Polar Field Models (Academic, New York, 1976).

${ }^{24}$ A. C. Eringen, J. Appl. Phys. 54, 4703 (1983).

${ }^{25}$ J. Peddieson, G. R. Buchanan, and R. P. McNitt, Int. J. Eng. Sci. 41, 305 (2003).

${ }^{26}$ L. J. Sudak, J. Appl. Phys. 94, 7281 (2003).

${ }^{27}$ Y. Q. Zhang, G. R. Liu, and J. S. Wang, Phys. Rev. B 70, 205430 (2004).

${ }^{28}$ Y. Q. Zhang, G. R. Liu, and X. Y. Xie, Phys. Rev. B 71, 195404 (2005).

${ }^{29}$ C. Sirtori, Nature (London) 417, 132 (2002).

${ }^{30}$ G. A. Antonelli and H. J. Maris, J. Appl. Phys. 91, 3261 (2002).

${ }^{31}$ E. B. Brauns, Phys. Rev. Lett. 88, 158101 (2002).

${ }^{32}$ J. Yoon, C. Q. Ru, and A. Mioduchowski, J. Appl. Phys. 93, 4801 (2003).

${ }^{33}$ J. Yoon, C. Q. Ru, and A. Mioduchowski, Composites, Part B 35, 87 (2004).

${ }^{34}$ S. P. Timoshenko, Philos. Mag. 41, 744 (1921).

${ }^{35}$ Q. Wang and V. K. Varadan, Int. J. Solids Struct. 43, 254 (2006). 